\title{
Propuesta metodológica para medir la pertinencia y el impacto de programas de pregrado en ingeniería
}

\author{
Nancy Elena Hamid-Betancur, Johny Álvarez-Salazar \& María C. Torres-Madroñero \\ Facultad de Ingenierías, Instituto Tecnológico Metropolitano, Medellín, Colombia.nancyhamid@itm.edu.co,.johnyalvarez@itm.edu.co, \\ mariatorres@itm.edu.co
}

\begin{abstract}
Resumen- En los lineamientos del Consejo Nacional de Acreditación para programas de pregrado del año 2013 se introducen nuevos aspectos a evaluar relacionados con estudios de pertinencia e impacto de los programas. Dada la creciente cultura de mejoramiento continuo, autoevaluación y acreditación que viene desarrollándose en las Instituciones de Educación Superior del país, nace la necesidad de dar respuesta a estos nuevos lineamientos. Este articulo propone una metodología para abordar los estudios de pertinencia e impacto para programas de pregrado en Ingeniería. La metodología propuesta consiste en tres etapas: definición del tipo de estudio, delimitación de la región de incidencia y desarrollo metodológico. Aunque la propuesta nace de las necesidades de los programas de pregrado de una Facultad de Ingenierías, en este artículo se describe una metodología flexible que puede ser adaptada a programas de pregrado y posgrado de diferentes áreas de conocimiento.
\end{abstract}

Palabras Clave - estudio de pertinencia, estudio de impacto, lineamientos de acreditación, CNA.

Recibido: 16 de mayo de 2017. Revisado: 30 de junio de 2017. Aceptado: 7 de julio de 2017.

\section{Methodological proposal to measure the pertinence and impact of engineering undergraduate programs}

Abstract - In 2013, the National Accreditation Council guideline for undergraduate programs introduced several new aspects to be evaluated related to pertinence and impact studies of undergraduate programs. Given the growing culture of continuous improvement, selfevaluation and accreditation that is being developed in the Higher Education Institutions of the country, there is a need to respond to these new guidelines. This article proposes a methodology to aboard the pertinence and impact studies for undergraduate programs in Engineering. The proposed methodology includes three stages: definition of type of study, delimitation of incidence region and the methodological development. Although the proposed methodology was born from the needs of undergraduate programs in a Faculty of Engineering, this article describes a flexible methodology that can be adapted to undergraduate and postgraduate programs of different areas of knowledge.

Keywords - pertinence study, impact study, accreditation guidelines, CNA.

\section{Introducción}

Para las Instituciones de Educación Superior (IES) en Colombia, las condiciones de alta calidad para la acreditación se refieren fundamentalmente a cómo una institución y sus programas orientan su deber ser hacia un ideal de excelencia, demostrando resultados específicos, tradición consolidada, impacto y reconocimiento social [1]. El Consejo Nacional de Acreditación CNA es el ente encargado de definir y reconocer la excelencia de los programas e instituciones [2]. Para lo cual propone los lineamientos tanto para acreditación Institucional como para los programas. Regularmente, estos lineamientos se usan a su vez para renovación del registro calificado y para acreditación de alta calidad. En el año 2013, el CNA establece nuevos lineamientos para la acreditación de programas de pregrado, Entre ellos se resaltan estudios de la relevancia académica y pertinencia social de los programas académicos. Ubicados en el Factor 1: Misión, proyecto institucional y de programa; Característica $\mathrm{N}^{\circ} 3$, relevancia académica y pertinencia social del programa: El programa es relevante académicamente $\mathrm{y}$ responde a necesidades locales, regionales, nacionales e internacionales [1].

Estos estudios han planteado un nuevo reto a las IES y programas académicos, dado que no existen directrices que orienten la formulación y desarrollo de los mismos. En virtud de lo anterior, se propone un modelo para evaluar la pertinencia e impacto social de los programas académicos de pregrado. El modelo se basa en estudios de tipo cerrado soportados a través de análisis estadístico. Para la elaboración se definió: El tipo de metodología, categorías, fuentes de información, criterio de ponderación de las categorías y de los análisis de los aspectos a evaluar. Adicionalmente, se propuso la matriz de ponderación, valoración e interpretación de acuerdo a la información encontrada.

En este artículo se presenta el modelo para la formulación de los estudios requeridos en los lineamientos de acreditación del CNA del año 2013. Diseñado para programas de ingeniería, sin embargo, es flexible para ser aplicado a cualquier área de conocimiento. En la sección 2 se presenta la propuesta metodológica para los estudios de pertinencia e impacto del programa, la sección 3 muestra un caso de estudio como propuesta de ponderación, y en la sección 4 se

Como citar este artículo: Hamid-Betancur, N.E., Alvarez-Salazar, J. and Torres-Madronero, M.C., Propuesta metodológica para medir la pertinencia y el impacto de programas de pregrado en ingeniería. Educación en Ingeniería, 12(24), pp. 97-100, Julio, 2017. 
presentan conclusiones y recomendaciones para la utilización de esta metodología.

\section{Propuesta metodológica}

El diseño de la metodología para el estudio de pertinencia e impacto de programas consiste en tres etapas. La primera busca definir el tipo de estudio, la segunda delimita la región de estudio y por último propone el desarrollo metodológico.

De acuerdo a Flores-Arce et al. [3], el tipo de estudio se define una vez se tienen claros los objetivos. Los tipos de estudio pueden ser cerrados o abiertos. Los estudios tipo cerrado están enfocados en determinar la pertinencia de una opción de formación para nueva creación o para su modificación o actualización. Por su parte, el estudio de tipo abierto parte de una región previamente seleccionada donde se busca identificar nuevas necesidades u opciones de formación pertinentes [3]. Por tanto, para el caso de estudios de pertinencia e impacto de programas se recomienda un estudio de tipo cerrado, dado que partimos de programas ya establecidos que se están autoevaluando para procesos de acreditación y mejoramiento continuo.

Para la delimitación de la región de estudio se recomienda emplear la región de incidencia de la IES, es decir, la región donde se encuentra la población estudiantil del programa. Por ejemplo, para el caso de los programas de pregrado del Instituto Tecnológico Metropolitano se establece como región de estudio el área metropolitana del Valle de Aburra, región de influencia de la Institución.

Finalmente, se define la metodología a emplear para el desarrollo de los estudios de pertinencia e impacto de los programas. Para definir esta metodología es necesario (1) definir las variables que de acuerdo a los lineamientos del CNA se propone estudiar, (2) a partir de estas variables se establecen las fuentes de información y los instrumentos necesarios para realizar la búsqueda de información y/o recopilación de la misma, y (3) se establecen las políticas de valoración, ponderación e interpretación de las variables planteadas y de la información encontrada. A continuación, se describen cada una de estas etapas para el desarrollo de estudios de pertinencia e impacto de los programas académicos.

\subsection{Pertinencia del programa}

La pertinencia de los programas académicos se establece a partir de los estudios requeridos por el CNA en los lineamientos de programas de pregrado de 2013. De acuerdo a los aspectos a evaluar relacionados con los estudios se determinó si apuntaba a la pertinencia o al impacto del programa. Una vez categorizados los estudios se definieron las fuentes de información para cada estudio.

\subsubsection{Descripción del estudio de pertinencia, categorías y aspectos a analizar}

La pertinencia del programa se aborda desde los siguientes cuatro estudios: 1) estudios orientados a identificar las necesidades y requerimientos del entorno laboral (local, regional y nacional) en términos productivos y de competitividad, tecnológicos y de talento humano y las acciones del programa para atenderlos; 2) estudios que demuestren la necesidad social del programa en la metodología que se ofrece; 3) estudios actualizados sobre las necesidades formativas en la región de influencia del programa; y 4) estudios y/o proyectos formulados o en desarrollo, que propendan por la modernización, actualización y pertinencia del currículo de acuerdo con las necesidades del entorno.

El primer estudio se ubica en la categoría de relevancia académica del programa (categoría 1), enfocada en las tendencias de las líneas de desarrollo afines al programa y los requerimientos del entorno empresarial en los ámbitos local, regional y nacional. En primera instancia, se realiza el análisis de las necesidades y tendencias de desarrollo en el entorno empresarial y en los ámbitos local, regional y nacional, identificando las necesidades desde la percepción de los empresarios y la oferta académica en el contexto nacional e internacional.

El segundo estudio se ubica en la categoría de flexibilidad metodológica del programa (categoría 2) enfocado en los requerimientos del entorno y en el análisis de las metodologías de enseñanza-aprendizaje. Se parte de analizar las diferentes razones sociales que demuestren la necesidad de la metodología ofrecida, y posteriormente, se analiza las metodologías de enseñanzaaprendizaje, tales como virtual, formación por competencias, ciclos propedéuticos, flexibilidad horaria, entre otros.

El tercer estudio se ubica en la categoría de necesidad formativa en la región (categoría 3), que parte de la identificación de las necesidades formativas y demanda de profesionales en la región de incidencia de la Institución.

El cuarto estudio se ubica en la categoría de pertinencia social del programa (categoría 4), enfocada en dos aspectos: calidad académica y contribución. En esta categoría se analiza los pensum de los programas por medio de los egresados y se determina el aporte del programa a la formación profesional y al proyecto de vida del egresado.

\subsubsection{Fuentes de información para dar respuesta a los estudios de pertinencia}

Las fuentes que se pueden tener en cuenta para el análisis de los estudios de pertinencia pueden ser los siguientes: el estudio de factibilidad del programa, encuestas a empresarios, cartas de los empresarios donde especifican la necesidad de practicantes, información gremial, documento maestro, sistemas de información estadísticos de la institución, encuestas de aspirante a ingresar a la educación superior, informe diagnóstico de causas de deserción, informe de caracterización de la trayectoria laboral de los egresados, planes de desarrollo nacional, local e institucional, clusters, entre otros.

\subsection{Impacto del programa}

\subsubsection{Descripción del estudio de impacto, categorías y aspectos a analizar}

El impacto del programa se aborda desde el estudio orientado a evaluar el impacto del programa con respecto al cumplimiento de sus propósitos y objetivos, así como la incidencia en el entorno social y su grupo de referencia disciplinar o profesional (estudio cinco). Este se dividió en dos categorías: impacto de los egresados en el medio (categoría 5) e impacto del programa visto desde lo social (categoría 6). 
El impacto de los egresados en el medio se enfoca desde la trayectoria laboral, teniendo en cuenta la situación laboral actual del egresado y el nivel de empleabilidad acorde al observatorio laboral, y desde el impacto mismo del egresado en el medio, analizado desde el desarrollo de las competencias del egresado y la correspondencia entre perfil profesional y ocupacional.

El impacto del programa visto desde lo social considera el desarrollo y calidad de vida de los estudiantes al terminar su proceso de formación y entrar a hacer parte de la comunidad como egresados del programa, teniendo en cuenta la percepción de egresados y su el informe de caracterización de la trayectoria laboral de los egresados.

\subsubsection{Fuentes de información para dar respuesta a los estudios de impacto}

Las fuentes que se pueden tener en cuenta para el análisis de los estudios de impacto pueden ser: informe de caracterización trayectoria laboral de los egresados, informe del observatorio laboral, encuestas a egresados y empresarios, proyectos generados desde extensión e investigación, clasificación de Colciencias para los grupos de investigación, entre otros.

\section{Caso de estudio: Propuesta de ponderación}

A partir de las categorías, aspectos analizados de cada una de ellas y fuentes de información, se asignó una ponderación, la cual resulta de una actividad de discusión académica con todos los integrantes y responsables de los procesos de autoevaluación de la Facultad. A continuación, se presenta una propuesta de ponderación para programas de ingeniería. El ejercicio académico acordó los porcentajes para cada uno de los ítems, partiendo de la categoría y hasta llegar a las fuentes de información

\subsection{Pertinencia social}

La pertinencia social es definida en [4] como el grado de correspondencia que debe existir entre las necesidades sociales e individuales que se pretende satisfacer con la educación universitaria y lo que realmente se llega a alcanzar. Después de la discusión académica se le asignó una ponderación del 30\% a las categorías de relevancia académica y pertinencia social del programa, porque se consideró que estas categorías evidencian las necesidades que requiere el medio. Mientras que a las categorías 2 y 3 se les asigno una ponderación del $20 \%$, ya que están más relacionadas con la metodología de enseñanza y la flexibilidad. Se presenta en la Tabla 1 las primeras cuatro categorías del estudio, enfocadas en la pertinencia del programa, con la ponderación respectiva de las fuentes de información y su análisis.

\subsection{Impacto}

El impacto puede plantearse de acuerdo a Martinez de Carrasquero et al. [5] como la responsabilidad social universitaria vinculada con el entorno. De ahí que se tiene en cuenta en porcentajes iguales el impacto de los egresados en el medio y el impacto del programa visto desde lo social. Se presenta en la Tabla 2 las categorías 5 y 6 con los ítems propuestos a analizar y sus respectivas fuentes de comparación y consulta.

\section{Conclusiones y recomendaciones}

La propuesta del modelo para medir la pertinencia e impacto de programas de pregrado en ingeniería permite analizar, no solamente la responsabilidad académica y profesional, sino también la responsabilidad social con que se está asumiendo la educación superior. De esta forma se puede implementar acciones de mejora, para corregir las falencias y debilidades encontradas en los aspectos evaluados en cada una de las categorías del modelo.

La metodología propuesta para adelantar los estudios se basa en definir las fuentes de información. Estas se convierten en el punto de partida para definir los indicadores de cumplimiento en cuanto a la pertinencia e impacto de los programas académicos. Y por ende en la herramienta de retroalimentación para apuntarle siempre al mejoramiento continuo de una forma asertiva.

Tabla 1.

Pertinencia del programa

\begin{tabular}{|c|c|c|c|}
\hline \multicolumn{4}{|l|}{ Categoría 1: Relevancia académica } \\
\hline Análisis & $\%$ & Fuente & $\%$ \\
\hline \multirow{4}{*}{$\begin{array}{l}\text { Análisis de las necesidades y } \\
\text { tendencias de desarrollo en el } \\
\text { entorno empresarial y en los } \\
\text { ámbitos local, regional y nacional }\end{array}$} & \multirow{4}{*}{15} & Estudio de factibilidad & 3 \\
\hline & & Encuestas empresarios & \\
\hline & & $\begin{array}{l}\text { Cartas de empresas } \\
\text { solicitando practicantes }\end{array}$ & 6 \\
\hline & & Información gremial & 6 \\
\hline $\begin{array}{l}\text { Identificación de las necesidades } \\
\text { desde la percepción de los } \\
\text { empresarios. }\end{array}$ & 3 & Encuestas Empresarios & 3 \\
\hline \multirow[t]{2}{*}{$\begin{array}{l}\text { Oferta académica en el contexto } \\
\text { nacional e internacional }\end{array}$} & 12 & $\begin{array}{l}\text { Documento maestro. } \\
\text { Sistemas de información. }\end{array}$ & 12 \\
\hline & & Ponderación categoría & 30 \\
\hline \multicolumn{4}{|c|}{ Categoría 2: Flexibilidad metodológica } \\
\hline Análisis & $\%$ & Fuente & $\%$ \\
\hline & Encuesta & \\
\hline $\begin{array}{l}\text { sociales que demuestren la } \\
\text { necesidad de la metodología } \\
\text { ofrecida }\end{array}$ & 7 & $\begin{array}{l}\text { Informe diagnóstico de } \\
\text { causas de deserción } \\
\text { presentado por SIGA }\end{array}$ & 7 \\
\hline \multirow[t]{2}{*}{$\begin{array}{l}\text { Análisis de las metodologías de } \\
\text { Enseñanza-Aprendizaje (Virtual, } \\
\text { formación por competencias, ciclos } \\
\text { propedéuticos, flexibilidad horaria, } \\
\text { entre otros). }\end{array}$} & \multirow[t]{2}{*}{13} & $\begin{array}{l}\text { Informe Caracterización } \\
\text { Trayectoria Laboral de } \\
\text { los Egresados. } \\
\text { Planes de Desarrollo. } \\
\text { Conpes. } \\
\text { Plan CT+I de Medellín. } \\
\text { Ruta N. } \\
\text { Cluster. } \\
\text { Mesas de trabajo Sena }\end{array}$ & 13 \\
\hline & & Ponderación categoría & 20 \\
\hline \multicolumn{4}{|c|}{ Categoría 3: Necesidad formativa en la región } \\
\hline Análisis & $\%$ & Fuente & $\%$ \\
\hline \multirow[t]{2}{*}{$\begin{array}{l}\text { Identificación de las necesidades } \\
\text { formativas en la región }\end{array}$} & \multirow[t]{2}{*}{20} & $\begin{array}{l}\text { Planes de Desarrollo. } \\
\text { Conpes. } \\
\text { Plan CT+I de Medellín. } \\
\text { Ruta N. } \\
\text { Cluster. } \\
\text { Mesas de trabajo Sena. }\end{array}$ & 20 \\
\hline & & Ponderación categoría & 20 \\
\hline \multicolumn{4}{|c|}{ Categoría 4: Pertinencia social del programa } \\
\hline Análisis & $\%$ & Fuente & $\%$ \\
\hline $\begin{array}{l}\text { Análisis de los pensum de los } \\
\text { programas por medio de los } \\
\text { egresados }\end{array}$ & 18 & \begin{tabular}{lr}
\multicolumn{2}{l}{ Documento Maestro. } \\
Informe & Trayectoria \\
Laboral vs & SIA (Año \\
graduación) &
\end{tabular} & 18 \\
\hline \multirow[t]{2}{*}{$\begin{array}{l}\text { Contribución del programa a la } \\
\text { formación profesional, al proyecto } \\
\text { de vida del egresado }\end{array}$} & 12 & Encuesta de egresados & 12 \\
\hline & & Ponderación categoría & 30 \\
\hline
\end{tabular}

Fuente: Autores. 
Tabla 2

Impacto del programa

\begin{tabular}{|c|c|c|c|}
\hline \multicolumn{4}{|c|}{ Categoría 5: Impacto de los egresados en el medio } \\
\hline Análisis & $\%$ & Fuente & $\%$ \\
\hline $\begin{array}{l}\text { Situación Actual Laboral del } \\
\text { Egresado. }\end{array}$ & 18 & $\begin{array}{l}\text { Informe } \\
\text { Caracterización } \\
\text { Trayectoria Laboral de } \\
\text { los Egresados. }\end{array}$ & 18 \\
\hline $\begin{array}{l}\text { Nivel de empleabilidad acorde } \\
\text { al observatorio laboral. }\end{array}$ & 12 & Observatorio Laboral & 12 \\
\hline $\begin{array}{l}\text { Desarrollo de las competencias } \\
\text { del egresado en el medio. }\end{array}$ & 10 & $\begin{array}{l}\text { Encuesta egresados y } \\
\text { empresarios. }\end{array}$ & 10 \\
\hline $\begin{array}{l}\text { Correspondencia entre perfil } \\
\text { profesional y ocupacional. }\end{array}$ & 10 & $\begin{array}{l}\text { Encuesta egresados y } \\
\text { empresarios. }\end{array}$ & 10 \\
\hline & & Ponderación categoría & 50 \\
\hline \multicolumn{4}{|c|}{ Categoría 6: Impacto del programa visto desde lo social } \\
\hline Análisis & $\%$ & Fuente & $\%$ \\
\hline $\begin{array}{l}\text { Mejoras de las condiciones } \\
\text { socioeconómicas (SIA vs } \\
\text { Trayectoria laboral). }\end{array}$ & 25 & $\begin{array}{l}\text { Salario egresado } \\
\text { Salario antes de } \\
\text { ingreso al ITM. } \\
\mathrm{N}^{\circ} \text { de egresados han } \\
\text { creado empresa y/0 } \\
\text { trabajo independiente } \\
\text { área formación. }\end{array}$ & 25 \\
\hline $\begin{array}{l}\text { Forma como se impacta desde } \\
\text { proyectos de extensión o } \\
\text { Investigación. }\end{array}$ & 15 & $\begin{array}{l}\mathrm{N}^{\circ} \text { Proyectos de } \\
\text { investigación/extensió } \\
\mathrm{n} \quad \text { ejecutados del } \\
\text { programa. } \\
\text { Clasificación } \\
\text { Colciencias }\end{array}$ & 15 \\
\hline \multirow[t]{2}{*}{$\begin{array}{l}\text { Impacto del programa en cuanto } \\
\text { a sus egresados y su desempeño }\end{array}$} & 10 & $\begin{array}{l}\text { Encuesta egresados y } \\
\text { empresarios }\end{array}$ & 10 \\
\hline & & Ponderación categoría & 50 \\
\hline
\end{tabular}

Fuente: Autores.

La metodología descrita en este artículo puede ser usada para programas en otros campos del conocimiento, teniendo en cuenta la descripción de cada categoría. Por lo tanto, se presenta una herramienta de base para dar respuesta a los aspectos a evaluar relacionados con estudios, introducidos en los lineamientos del CNA para programas de pregrado en el 2013.

\section{Referencias}

[1] Lineamientos para la acreditación de programas de pregrado. CNA, Consejo Nacional de Acreditación. Colombia, 2013.

[2] Hamid, N.E. and Torres-Madronero, M.C., Acreditación de programas en ingeniería en la región: Análisis comparativo, Revista Educación en Ingeniería, 10(19), pp. 80-89, 2015.

[3] Flores-Arce, L.C., González-Basilio, S., Siordia-Medina, P.R. and ZeaVerdín, A.A., Propuesta metodológica para la realización de estudios de pertinencia, Secretaría de Docencia, Universidad Autónoma de Nayarit, Nayarit, México, nov. 2010

[4] La educación superior en el siglo XXI: Líneas estratégicas de desarrollo, una propuesta de la ANUIES. Asociación Nacional de Universidades e Instituciones de Educación Superior ANUIS, México, 2000.

[5] Martínez-de Carrasquero, C., Mavpárez, R., Rojas, P., Ligibther, A. and Carvallo, B., La responsabilidad social universitaria como estrategia de vinculación con su entorno social, Frónesis, 15(3), pp. 81-103, 2008.
N.E. Hamid-Betancur, recibió el título de Ing. de Sistemas en 1994 de la Universidad San Buenaventura, sede Medellín, el título de Esp. en la Administración de la Informática Educativa en 2013, y el título de MSc. en Gestión de la Tecnología Educativa en 2015 de la Universidad de Santander, Colombia. Se vinculó al Instituto Tecnológico Metropolitano desde 2013 como docente ocasional de tiempo completo, en el Departamento de Electrónica y Telecomunicaciones de la Facultad de Ingenierías. Desde el agosto de 2013 hasta la actualidad es la líder de la Unidad de Autoevaluación de la Facultad de Ingenierías.

ORCID: 0000-0002-0311-1414

J. Alvarez-Salazar, recibe el título de Ing. Electromecánico en 2009, el título de MSc. en Automatización y Control Industrial en 2014, ambos del Instituto Tecnológico Metropolitano, Medellín, Colombia. Ha trabajado en programas y proyectos del área de automatización y control, con énfasis en control lógico programable y desde 2008 trabaja en el Instituto Tecnológico Metropolitano, sede Medellín en el área de automatización industrial.

ORCID: 0000-0002-7041-8619

M.C. Torres-Madroñero, recibió el título de Ing. Electrónica en 2006 de la Universidad Nacional de Colombia, sede Manizales, el título de MSc. en Ingeniería Eléctrica en 2008 y su PhD. en Ciencias e Ingeniería de la Computación y la Información en 2013, ambos de la Universidad de Puerto Rico, Recinto Universitario de Mayagüez. Se vinculó como docente de carrera en el Instituto Tecnológico Metropolitano de Medellín desde 2015. Para el 2017, es profesora asistente del Departamento de Sistemas de Información de la Facultad de Ingenierías, líder del grupo de investigación en Automática, Electrónica y Ciencias Computacionales, e investigadora de la línea de máquinas inteligentes y reconocimiento de patrones - MIRP.

ORCID: 0000-0002-9795-2459 